\title{
Calidad de plántulas de tres especies forrajeras (Moringa oleifera Lam., Leucaena leucocephala y Cajanus cajan) en condiciones de vivero
}

\section{Seedling quality forage (Moringa oleifera Lam., Leucaena Leucocephala and Cajanus cajan) in nursery conditions}

\begin{abstract}
Álvaro Noguera-Talavera ${ }^{1}$, Nadir Reyes-Sánchez ${ }^{2}$, Juan José Membreño ${ }^{1}$, Carlos Duarte-Aguilar ${ }^{3}$, Bryan Mendieta-Araica ${ }^{2}$. ${ }^{1}$ Docentes investigadores, Facultad de Recursos Naturales y del Ambiente / 2 Docentes investigadores, Facultad de Ciencia Animal /

${ }^{3}$ Ing. en Recursos Naturales / (Para correspondencia: alvaro.noguera@ci.una.edu.ni)
\end{abstract}

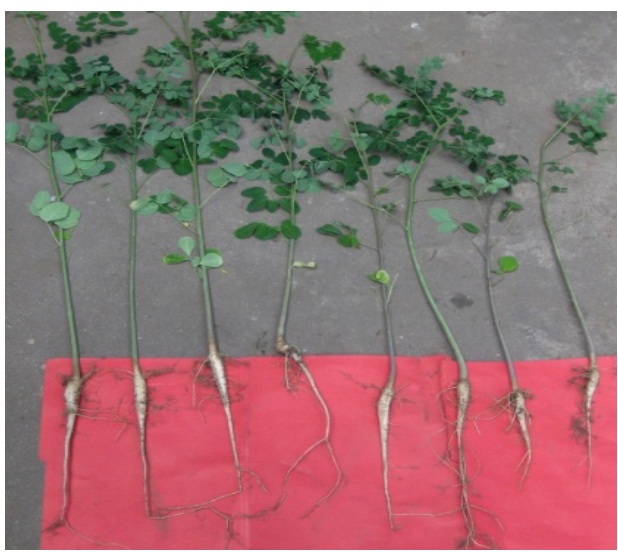

\section{RESUMEN}

Con el objetivo de caracterizar el comportamiento inicial de $\mathrm{Mo}$ ringa oleifera y profundizar en las ventajas de esta especie, en comparación con otras forrajeras de rápido crecimiento (Leucaena leucocephala y Cajanus cajan); se realizó en el vivero de la Facultad de Recursos Naturales y del Ambiente, un ensayo en diseño completamente aleatorio con 20 repeticiones por especie. Se realizó un conteo diario de germinación por un periodo de tres semanas (21 días); mientras patrones de crecimiento fueron determinados a partir de evaluaciones semanales de diámetro basal y raíz, longitud de tallo y raíz, y número de raíces secundarias; así como producción de biomasa expresada en peso seco y peso fresco de la parte área y radicular, en muestra de ocho plantas destruidas semanalmente. La comparación en el desempeño de cada especie fue contrastada mediante el uso de índices de calidad de plantas. Los resultados muestran ventajas de la especie $M$. oleifera para la mayoría de variables; solo superado en el número de raíces secundarias por las dos especies en estudio. Los índices de calidad sugieren pocos problemas de establecimiento en campo para las especies $M$. oleifera y L. leucocephala como producto del rápido crecimiento, y aun con la desigual proporción en la relación tallo-raíz, y biomasa. Los valores de calidad registrados para cada especie pueden ser modificados a través de prácticas silviculturales en las plantas en el vivero.
ABSTRACT
With the objective to determinate the initial behavior of Moringa oleifera, and deep into advantage of this specie in comparison to others fodder with quick growth (Leucaena leucocephala and Cajanus cajan); was carried out an experiment with random complete design with, and 20 samples. By a period of three weeks (21 days) was monitored the germination; while patterns of growth were determined through evaluation for ten weeks of variables of diameter and root, long stem and root, and secondary root numbers, also biomass production showed as dry and fresh matter of root and, foliage and branches parts of eight plants were destroyed weekly. The quality of plants was compared using quality indexes based on morphological variables. The results suggest a best behavior of $M$. oleifera to almost all variables, having best performance $C$. cajan and L. leucocephala in secondary root numbers. The quality indexes suggest few problems of establishment on field to the species $M$. oleifera and L. leucocephala, this due to the quick growth even with unequal proportion on stem-root growth relation and biomass production. The quality index values obtained to each species could be modified through silvicultural management of the plant at the nursery. 
$\mathrm{E}$ 1 establecimiento con éxito de una plantación es el principal objetivo en el proceso de repoblación forestal (Birchler et al., 1998) tanto para fines de rehabilitación del suelo, como de producción de bienes como forraje, semillas, extractos, entre otros. Algunos impedimentos encontrados en el cumplimiento de este objetivo son: la existencia de factores micro climáticos específicos del lugar de plantación y potencialmente perjudiciales para la misma, la competencia vegetal, los daños producidos por animales, el uso de procedimientos de plantación incorrectos, o el empleo de planta fisiológicamente inadecuada (Birchler et al., 1998).

Los viveros forestales tratan de producir planta de la mayor calidad y de la forma más eficiente posible desde un punto de vista económico. Esto implica la definición de planta de calidad, así como la mejor manera de evaluarla, aspectos que siguen siendo objeto de continuo debate e investigación (Birchler et al., 1998).

Moringa oleifera Lam., conocido comúnmente como marango y nativa de la India y Pakistán, está siendo ampliamente distribuida alrededor del mundo, especialmente a través de la zona tropical (Reyes-Sánchez et al., 2006).

De acuerdo con Nouman et al., (2012) Moringa es una especie que se propaga fácilmente a través de semillas y por medio de tallos. Este autor refiere que indudablemente la propagación vegetativa de marango es fácil pero, para que la producción de biomasa para forraje en sistemas ganaderos sea eficiente, se requiere la propagación a través de semillas.

Según Nouman et al., (2012) en ensayos con marango se ha documentado porcentajes de germinación distribuidos en un rango entre $60 \%$ y $90 \%$ con el uso de semillas frescas, y un periodo de germinación que va de siete a 30 días después de la siembra. Sin embargo, un problema identificado por este autor es la pérdida de viabilidad de la semilla de $60 \%$, 48\% y 7.5\% después de dos a cuatro meses de la recolección; al respecto se hace referencia que semillas viejas tienen problemas de germinación debido a su alto contenido de aceite y ataque de insectos.

Aun con el creciente auge como cultivo, existe poca información sobre el comportamiento de Moringa oleifera en sus primeras etapas de desarrollo, por lo que el objetivo de esta investigación fue caracterizar plantas en condiciones de vivero y profundizar en las posibles variaciones entre especies, al comparar el desarrollo inicial de marango con otras forrajeras de rápido crecimiento como Leucaena leucocephala y Cajanus cajan.

\section{MATERIALES Y MÉTODOS}

Localización del área de estudio. El ensayo se estableció en el vivero de la Facultad de Recursos Naturales y del Ambiente de la Universidad Nacional Agraria (UNA) ubicado en el km 12 1/2 Carretera Norte en el municipio de Managua. Esta zona está localizada en el litoral central del pacífico entre las coordenadas geográficas de $12^{\circ} 08^{\prime}$ de latitud norte y $86^{\circ} 10^{\prime}$ de longitud oeste, a una altura de $56 \mathrm{msnm}$. Las condiciones climáticas del área experimental corresponden a una zona de vida ecológica de bosque tropical seco, con temperatura media anual de $27.3^{\circ} \mathrm{C}$, humedad relativa media anual de $73.2 \%$, y una precipitación media anual de $1264.2 \mathrm{~mm}$ (INETER, 2006).

Tratamientos. El procedimiento definido para este estudio, se refiere a la comparación de $M$. oleífera, con especies de L. leucocephala, y C. cajan, por tener como patrón común su utilización en sistemas agropecuarios, rápido crecimiento e importante grado de adaptación.

Característica del sustrato utilizado. El sustrato utilizado fue $100 \%$ suelo con el que se llenaron bolsas de polietileno. El análisis químico del sustrato indica los siguientes niveles: $\mathrm{N}=$ $0.06 \%$, en el caso del $\mathrm{K}$, Ca y Mg se expresan en meq/100 g de suelo y el fósforo $(\mathrm{P})$ y los micronutrientes en ppm (partes por millón), siendo los valores para $\mathrm{K}=1.70, \mathrm{Ca}=18.48$ y $\mathrm{Mg}=$ $5.40 ; \mathrm{P}=7.51, \mathrm{Fe}=31.84, \mathrm{Cu}=8.37, \mathrm{Mn}=11.63 \mathrm{y} \mathrm{Zn}=3.32$.

Procedimiento experimental. El origen de la semilla fue diferente para cada especie, en el caso de Leucaena fue obtenida en el banco de semillas del Instituto Nacional Forestal, correspondiendo a un lote con numeración I. 17263; la semilla de la especie Marango fue colectada en un área de banco forrajero localizado en la finca Santa Rosa en la Universidad Nacional Agraria; mientras la semilla de Gandul fue provista por el centro experimental del Instituto Nicaragüense de Tecnología Agropecuaria.

Previo a la siembra se realizó una selección de semillas, utilizando como criterios el tamaño y la calidad potencial basada en la ausencia de daños por insectos, principalmente orificios. Así mismo, se realizó un proceso de raspado de la testa de la semilla como tratamiento de escarificación. En cada bolsa se colocaron dos semillas de cada especie. Tanto las semillas como las plantas recibieron riego diario (un litro) por la mañana y por la tarde usando una regadera.

Diseño experimental. Se utilizó un diseño completamente aleatorio (DCA), con 20 repeticiones por especies. El orden en que fueron constituidos los bloques fue de manera aleatoria, siendo la secuencia marango, leucaena y gandul.

Mediciones. Se realizó un conteo diario de germinación por un periodo de tres semanas, obteniendo así los datos del número de emergencia por día y por especie.

En el caso de las variables altura de planta y diámetro basal se realizaron con frecuencia semanal en 20 plantas, iniciando siete días después de la siembra, y de manera paralela haciendo muestreos destructivos en ocho plantas por medición durante 10 semanas. 
Las variables evaluadas fueron: altura de planta o longitud del tallo en centímetros (con regla graduada, desde el nivel del suelo hasta el ápice de la rama apical), diámetro del tallo en centímetros (en la base de la plántula, con pie de rey), número de raíces secundarias (mediante conteo visual), longitud de la raíz (en centímetros, con regla graduada), y diámetro de la raíz (en el caso de marango el dato anotado fue el promedio obtenido al medir el diámetro en la parte inmediata a la unión con el tallo, al centro de la raíz y próximo a la cofia; esto debido a la morfología de la raíz de esta especie).

De manera complementaria, se realizó el cálculo de peso fresco y peso seco de la parte aérea y de la fracción radicular usando una balanza electrónica de precisión. La obtención del valor de peso seco se registró posterior al secado en horno durante 24 horas a temperatura de $55^{\circ} \mathrm{C}$.

De los valores de las variables morfológicas se realizó el cálculo de índices de calidad de plantas, siendo estos el índice de robustez (IR), índice de calidad de Dickson (ICD), coeficiente de altura del tallo y longitud de raíz (AT/LR), índice de lignificación (IL) y el coeficiente de biomasa seca aérea y biomasa seca de raíz (BSA/BSR); estos como parámetros de decisión para determinar el momento más apropiado de traslado de las plantas (principalmente de marango) al campo.

Los datos fueron procesados y analizados con el programa Infostat, realizando el cálculo de estadísticos descriptivos, análisis de varianza y correlación entre variables.

\section{RESULTADOS Y DISCUSIÓN}

Porcentajes de germinación marcadamente diferentes fueron registrados entre especies; dándose el valor más alto para M. oleifera con 98\%; contrario a $L$. leucocephala y $C$. cajan, cuyos valores fueron $71 \%$ y $17 \%$ respectivamente.

El resultado obtenido para marango, coincide con el valor reportado por González (2014) y Saavedra y Gutiérrez (2014) en ensayos cuyo sustrato correspondió a una mezcla de estiércol bovino y suelo, cien por ciento suelo común, y compost; así mismo Medina et al., (2007), reportan $100 \%$ de germinación en un estudio comparativo de marango con leucaena, registrando esta ultima un $95 \%$.

En relación al periodo de emergencia por especie, la figura 1 muestra un comportamiento igual en cuanto al día de inicio para las tres especies, ocurriendo al sexto día posterior a la siembra. El mayor número de emergencias al inicio correspondió a leucaena y gandul, sin embargo, a partir del séptimo día hasta el día nueve marango fue la especie con el mejor desempeño para este parámetro. El periodo de germinación de marango se extendió hasta el día 15, alcanzando 98\% de germinación.

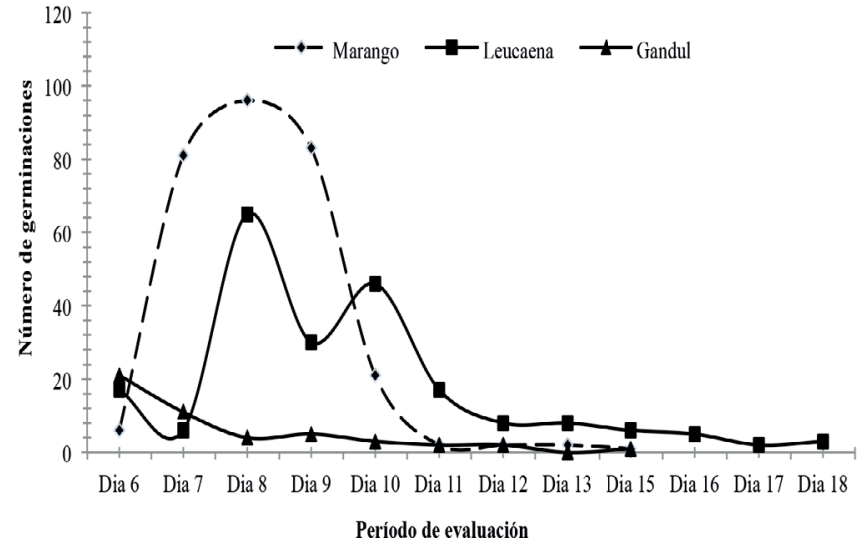

Figura 1. Comportamiento en la germinación de marango leucaena y gandul.

Los resultados relativos al desarrollo en altura muestran un crecimiento progresivo para las tres especies (figura 2) durante todo el periodo del ensayo. La mayor altura total para el periodo correspondió a marango $(70 \mathrm{~cm})$, que difiere y supera en mucho el valor reportado $(53.2 \mathrm{~cm})$ por $\mathrm{Me}-$ dina et al., (2007) en el mismo periodo de diez semanas.

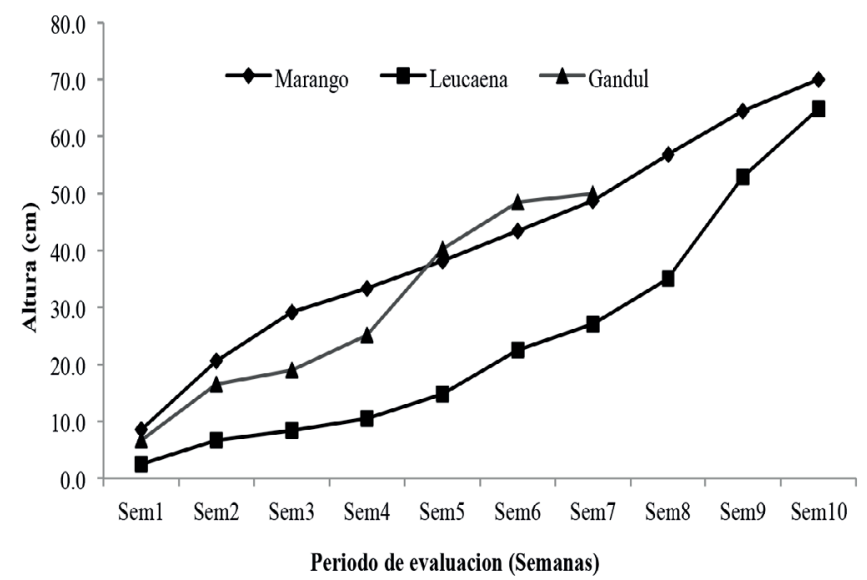

Figura 2. Altura de planta de marango, leucaena y gandul en etapa de vivero.

La tasa media de crecimiento en altura fue de $1 \mathrm{~cm}$ día $^{-1}$ para leucaena, mientras gandul y marango presentaron valores similares $\left(0.94\right.$ y $\left.0.92 \mathrm{~cm} \mathrm{día}^{-1}\right)$. No se registró diferencias estadísticamente $(p>0.05)$ entre especies.

La tasa de crecimiento más constante fue para marango y más significativo a partir de la quinta semana, al igual que gandul; mientras las mayores tasas de crecimiento para leucaena se dieron a partir de la octava semana.

Al igual que la altura, el crecimiento en diámetro fue progresivo para las tres especies, sin embargo, más discretos en comparación a la altura, registrándose diferencias significativas $(p<0.05)$ entre especies. 
Durante todo el ensayo la especie marango superó el crecimiento registrado por gandul y leucaena; alcanzando así el valor más alto en diámetro $(7.4 \mathrm{~mm})$ al final del ensayo. Los valores alcanzados por marango en este estudio difieren de los documentados por Medina et al., (2011) para el mismo periodo, en el cual el diámetro alcanzado fue de $9 \mathrm{~mm}$, y superior al diámetro de leucaena.

El patrón de crecimiento diamétrico en comparación a la altura puede explicarse a través de la teoría que establece que producto de la competencia entre individuos en el vivero, las plantas priorizan un mayor incremento en altura; por otro lado fisiológicamente es conocido que en las arbóreas el primer crecimiento se expresa en una continua elongación del tallo; mientras el aumento en diámetro es logrado por medio de movimiento de las plantas en el vivero; siendo esta una condición determinante para alcanzar una buena calidad de planta en la especie marango.

Un alto valor de correlación $(r=0.93)$ fue determinado entre el crecimiento en altura y el diámetro registrado para la especie marango.

En cuanto al comportamiento de las variables relacionadas a la parte subterránea, a través del muestreo destructivo se documentó los valores del número de raíces $(\mathrm{Nr})$, longitud de raíz principal (Lr) y diámetro de la raíz principal (Dr).

El parámetro número medio de raíces secundarias por medición fue diferente entre especies, sugiriendo mejor desempeño durante todo el estudio para marango, el que desde la primer semana mostró una mayor producción (cuadro 1) en comparación a leucaena y gandul, las cuales registraron valores relativamente similares durante la octava y sexta semana.

nes ambientales favorables para su crecimiento, emite nuevas raíces, las cuales iniciarán el proceso de absorción de agua; así mismo Davis y Jacobs, (2005) mencionan que el número de raíces secundarias de primer orden han mostrado correlación para mejorar el desempeño de las plantas en el campo.

La longitud de raíz experimentó un crecimiento progresivo, registrando valores similares entre especies con una ligera ventaja en la elongación de raíz para leucaena; en comparación a marango, el cual equiparó el crecimiento de leucaena a partir de la cuarta semana que coincide con el inicio de la forma definitiva (tuberosa) de la raíz de marango. El mismo periodo necesitó gandul para alcanzar el crecimiento radicular de leucaena.

En cuanto al diámetro de raíz es notorio el valor experimentado por marango, producto de la diferencia morfológica con las otras dos especies. En este sentido, el mayor crecimiento para marango inicia a partir de la quinta semana, posterior a la aparición definitiva (en la cuarta semana), de la raíz tuberosa típica de marango. Aun así, el patrón común entre las tres especies fue la velocidad de crecimiento durante el periodo de evaluación.

Calidad de planta de las especies en el ensayo Relación peso seco de la parte aérea y el peso seco del sistema radicular (BSA/BSR). Este parámetro como indicador del desarrollo de la planta y producción de biomasa en vivero fue significativamente diferente entre especies $(p<0.05)$. Como se indica en la figura 4, la especie con el valor más bajo fue marango, registrando una BSA/BSR 1.8 a la semana 10 , lo que indica que la biomasa aérea es mayor que la subterránea lo que según Rodríguez, (2008) es

Cuadro 1. Parámetros de la estructura radicular de tres especies forrajeras en etapa de vivero

\begin{tabular}{|c|c|c|c|c|c|c|c|c|c|}
\hline \multirow[t]{2}{*}{ Semana } & \multicolumn{3}{|c|}{ Marango } & \multicolumn{3}{|c|}{ Leucaena } & \multicolumn{3}{|c|}{ Gandul } \\
\hline & $\mathrm{Nr}$ & $\operatorname{Lr}(\mathrm{cm})$ & $\operatorname{Dr}(\mathrm{mm})$ & $\mathrm{Nr}$ & $\operatorname{Lr}(\mathrm{cm})$ & $\operatorname{Dr}(\mathrm{mm})$ & $\mathrm{Nr}$ & $\operatorname{Lr}(\mathrm{cm})$ & $\operatorname{Dr}(\mathrm{mm})$ \\
\hline 1 & 15 & 3.4 & 1.0 & 4 & 2.4 & 1.0 & 10 & 4.7 & 1.0 \\
\hline 2 & 11 & 6.2 & 1.0 & 3 & 10.8 & 1.5 & 13 & 6.1 & 1.0 \\
\hline 3 & 10 & 7.0 & 3.2 & 15 & 11.2 & 2.0 & 7 & 9.9 & 1.0 \\
\hline 4 & 15 & 12.0 & 3.8 & 12 & 14.5 & 2.8 & 10 & 12.8 & 1.5 \\
\hline 5 & 20 & 17.1 & 8.9 & 20 & 18.4 & 3.1 & 14 & 14.1 & 1.7 \\
\hline 6 & 35 & 19.7 & 11.7 & 16 & 23.8 & 4.0 & 55 & 27.1 & 2.0 \\
\hline 7 & 31 & 22.4 & 12.0 & 16 & 32.4 & 4.0 & 55 & 35.0 & 4.0 \\
\hline 8 & 42 & 26.4 & 12.3 & 25 & 34.9 & 4.0 & - & - & - \\
\hline 9 & 40 & 28.5 & 13.1 & 42 & 36.3 & 4.1 & - & - & - \\
\hline 10 & 33 & 36.7 & 14.9 & 67 & 37.8 & 5.1 & - & - & - \\
\hline
\end{tabular}

Nr: Número de raíz, Lr: Longitud de raíz principal, Dr: Diámetro de la raíz principal así, considerado una buena relación entre ambas partes; y sugiere la existencia de un sistema radical suficiente para proveer energía a la parte aérea de la planta.

El comportamiento expresado en los valores del indicador presentan una mayor variabilidad para las especies gandul y leucaena, no pudiendo determinarse la semana exacta en que la relación biomasa aérea $\mathrm{y}$ radical es la más apropiada para extraer la planta del vivero, no así en marango, que a partir de la quinta semana

Al respecto Sáenz, et al., (2010) y Davis y Jacobs, (2005) concluyen que la abundante emisión de raíces secundarias demuestra alta calidad y garantiza un rápido crecimiento después de la plantación; cuando se establece en condicio- muestra que la biomasa aérea es igual a la biomasa de la raíz; lo que combinado a otros indicadores puede darnos la idea del momento ideal para trasladar la planta del vivero a campo. 


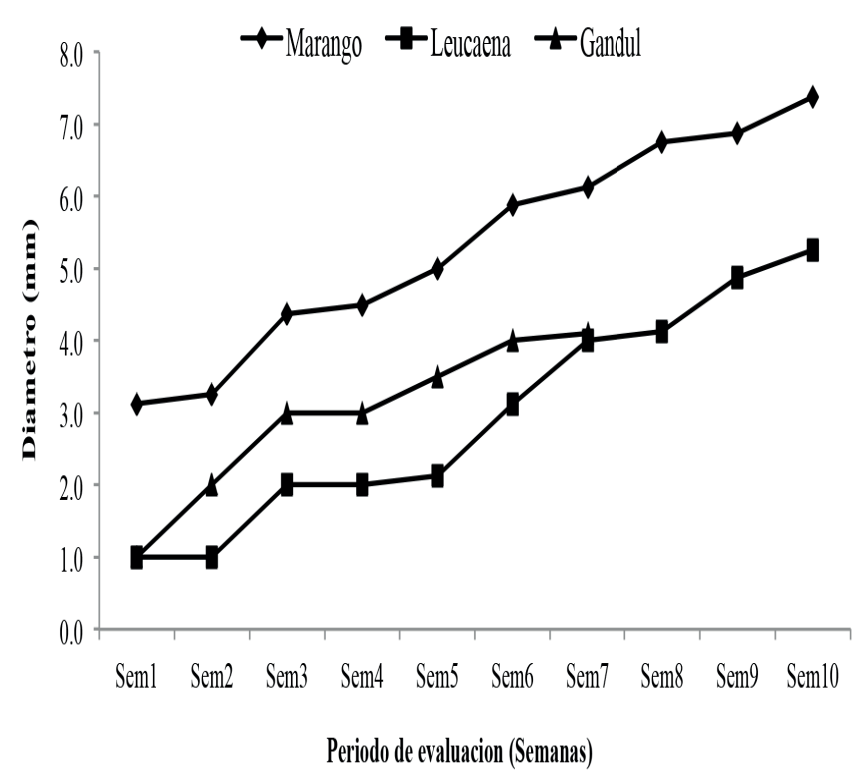

Figura 3. Crecimiento del diámetro basal de marango, leucaena y gandul en la fase de vivero.

En el caso de las especies leucaena y gandul por registrar valores superiores al rango establecido por Rodríguez (2008), -que debe fluctuar entre 1.5 y 2.5 - se presenta una desproporción en la relación biomasa aérea y biomasa radical; por tanto una raíz que no provee suficiente energía para una eficiente absorción de agua y nutrientes, ocurre un menor crecimiento del tallo una vez la planta sea llevada a campo, incrementándose el riesgo de una baja sobrevivencia.

El cociente tallo-raíz tiene como propósito, al igual que la mayoría de los índices determinar la proporción real entre el tallo y la raíz como predictor del éxito de las plantas una vez que son trasladas al campo; así los valores experimentados por las especies en el ensayo (Figura 5) muestran una adecuada proporción solamente para la especie leucaena desde el inicio de las mediciones hasta la octava semana, mientras el valor medio para esta especie fue de 0.97 .

Para el caso de gandul, este registró un valor aun permitido en la séptima semana y un valor medio de dos al final de la evaluación; mientras el comportamiento de marango fue de mejora progresiva en el valor del indicador a partir de la quinta semana, su mejor desempeño lo alcanzó en la décima semana; cuantificándose a la vez un valor medio de 2.5 que corresponde al valor máximo permitido para este indicador, pero esta proporción es solamente ideal para sitios de plantación sin limitante de humedad, lo que no es la realidad para los sitios en que se distribuye naturalmente o se recomienda plantar marango, planteando un reto para definir la proporción correcta.

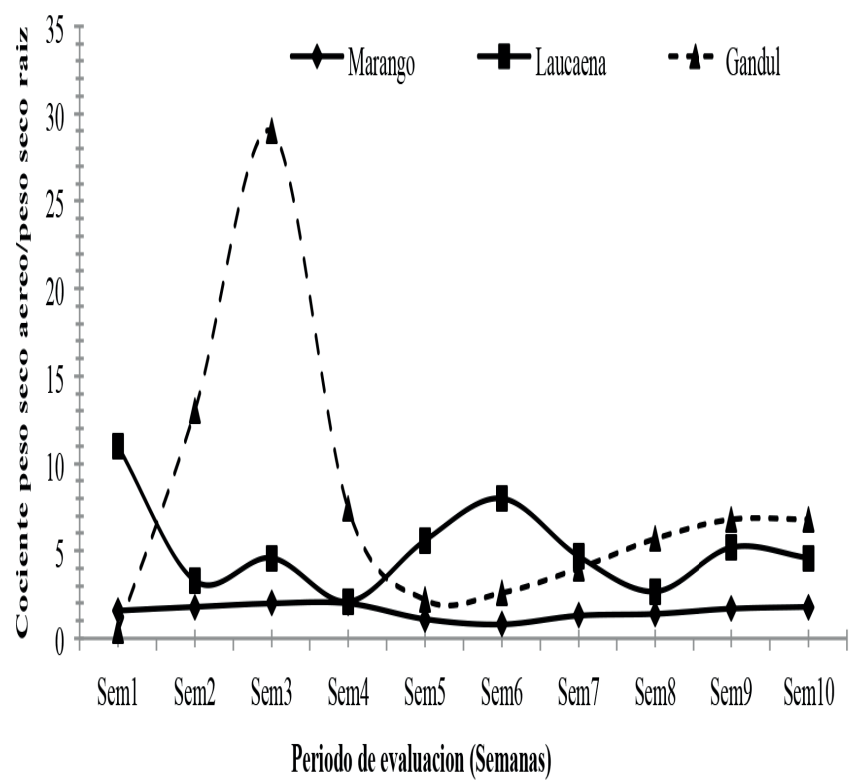

Figura 4. Cambios en la relación peso seco aéreo y peso seco de raíz en marango, leucaena y gandul en la fase de vivero.

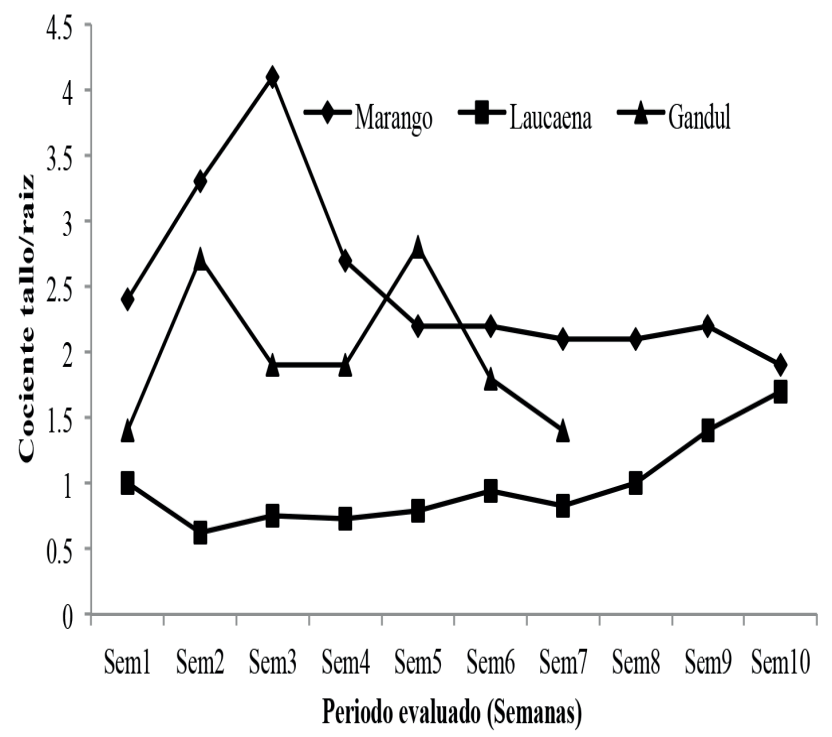

Figura 5. Cambios en la relación crecimiento de tallo y raíz en marango, leucaena y gandul en la fase de vivero.

Implicaciones del estudio. El comportamiento de las especies en el ensayo apunta hacia un mayor crecimiento tanto de la parte aérea como radical por parte de marango para la mayoría de las variables y parámetros evaluados, en comparación a las especies leucaena y gandul. 
De manera complementaria los indicadores de calidad cuantificados permiten definir con mayor certeza el potencial de sobrevivencia y establecimiento dentro de las áreas de plantación.

Los valores registrados para cada especie es comúnmente modificado a través de prácticas de manejo de las plantas en el vivero, sin embargo, la falta de información para la especie marango plantea la necesidad de inferir en los elementos a considerar para la obtención de plantas de calidad en un tiempo financieramente viable, $\mathrm{y}$ desde el punto de vista de la disminución de riesgo por pérdida en el campo.

En este sentido García (2007), indica que una planta de buena calidad debe presentar características como un diámetro basal grande, bajo valor del coeficiente de robustez, un sistema radicular fibroso y un bajo valor de la relación biomasa aérea y biomasa de raíz.

Un elemento de interés en cuanto a la relación entre la altura y el diámetro de plantas en este ensayo es el valor obtenido en el denominado en (Birchler et al., 1998) cociente de esbeltez o índice de robustez (IR) Sáenz et al., (2010), el que para las tres especies sugiere una baja equitatividad y por tanto baja robustez; ya que según los autores este debe ser menor de seis para indicar una alta resistencia a la desecación por el viento, y altas temperaturas asegurando una buena sobrevivencia y crecimiento en la plantación (cuadro 2).

En tal sentido, la especie gandul presentó el cociente más alto (9.3) y por tanto la menor esbeltez; mientras leucaena (7.1), y marango (7.3) registraron valores similares. Este comportamiento se constituyó en un patrón en las tres especies durante todo el periodo de evaluación, lo que indica que el rápido crecimiento en altura y un bajo ritmo de crecimiento en diámetro genera baja robustez en las plantas producidas en el vivero, pudiendo mejorarse esta condición mediante podas aéreas y movimiento de las plantas aun en las primeras tres semanas en el vivero. Orientando el análisis hacia una expresión más completa del equilibrio de la distribución de la masa y la robustez, evitando así seleccionar plantas desproporcionadas y descartar plantas de menor altura y poco vigor.

Sáenz et al., (2010), plantean el índice de calidad de Dickson el que para el presente ensayo fue registrado en 0.79 para marango, siendo significativamente diferente $(p<0.05)$ a los valores registrados por leucaena y gandul (cuadro 2). Al respecto, Sáenz et al., (2010) concluyen sobre la relación directa entre el valor del índice y la sobrevivencia de las plantas; así índice menor a 0.15 significa problemas en el establecimiento para algunas especies.
Cuadro 2. Índices de calidad de planta de tres especies forrajeras producidas en vivero

R: índice de robustez, ICD: índice de calidad de Dickson, AT/LR: coeficiente de altura
del tallo y longitud de raíz, IL: índice de lignificación, BSA/BSR: coeficiente de biomasa seca aérea y biomasa seca de raíz.

La baja relación entre el crecimiento en altura del tallo y la longitud de la raíz indica la necesidad de poda aérea al menos una semana antes de trasladar las plantas de marango al campo, o una combinación de poda con cambio de bancal dentro del vivero; lo que permitiría establecer una relación 0.8 a uno al inicio de la plantación y asegurar menos estrés y, una mayor sobrevivencia y establecimiento.

En índice de lignificación presenta bajos valores para las tres especies, lo que sugiere la necesidad de procesos de preparación de las plantas antes de salir del vivero, como comúnmente se da la disminución en la frecuencia de riego, fertilización y movimiento de la planta.

La utilidad de este indicador para la determinación de la calidad de plantas en marango, no se recomienda debido a que esta especie presenta un alto contenido de agua, expresada en un tallo notoriamente suculento, por lo que aun con medidas preparatorias antes de la salida del vivero posiblemente se alcancen bajos valores de lignificación.

\section{CONCLUSIÓN}

Las plántulas de marango producidas bajo las condiciones de este estudio, pueden extraerse del vivero y trasladarse al campo a partir de la quinta semana; pudiendo asegurarse un menor estrés y mortalidad en campo realizando un primer movimiento o cambio de bancal en la tercer semana; y un segundo movimiento y poda aérea en la cuarta semana. 


\section{REFERENCIAS BIBLIOGRÁFICAS}

Birchler, T; Rose, RW; Royo, A; Pardos, M. 1998. La planta ideal: revisión del concepto, parámetros definitorios e implementación práctica. Investigación agropecuaria. Vol. 7, No. 1 y 2. Pág. 109-121.

Davis, AS; Jacobs, DF. 2005. Quantifying root system quality of nursery seedlings and relationship to outplanting performance. New Forest. No. 30. p. 295-311.

García, MA. 2007. Importancia de la calidad del plantín forestal. XXII Jornadas Forestales de Entre Ríos. Área Forestal de la EEA Concordia del INTA. (en línea). Consultado 26 abr 2014. Disponible en http://www.inta.gov.ar/concordia/info/Forestales/ contenido/pdf/2007/312.II. GARCIA.pdf

González R, VA. 2014. Comportamiento de dos poblaciones de Moringa oleifera (material acriollado y mejorado PKM1) en sus primeras etapas de crecimiento en condiciones de vivero, UNA, Managua. Tesis de grado. Facultad de Ciencias Animales. Managua, NI. 29 p.

INETER (Instituto Nicaragüense de Estudios Territoriales). 2006. Informe meteorológico estación Aeropuerto internacional Augusto Cesar Sandino. Las Mercedes, Managua, NI. 134 p.

Medina, MG; García, DE; Moratinos, P; Cova, LJ. 2011. Comparación de tres leguminosas arbóreas sembradas en un sustrato alcalino durante el periodo de aviveramiento. I. Variables morfoestructurales. Pastos y forrajes, Vol. 34, No.1, p. 1-11.

Medina, MG; García, DE; Clavero, T; Iglesias, JM. 2007. Estudio comparativo Moringa oleifera y Leucaena leucocephala durante la germinación y la etapa inicial de crecimiento. Zootecnia tropical. 25(2). p. 83-93.

Nouman, W; Siddiqui, MT; Basra S, MA; Afzal, I; Rehman, H. 2012. Enhancement of emergence potential and stand establishment of Moringa oleifera Lam. by seed priming. Turkey Journal Agricultural and Forest . No. 36. p. 227-235

Reyes Sanchez. N; Ledin, S; Ledin, I. 2006. Biomass production and chemical composition of Moringa oleifera under different management regimes in Nicaragua. Agroforestry Systems. N. 66. p. 231-242.

Rodríguez T, DA. 2008. Indicadores de calidad de planta forestal. Universidad Autónoma Chapingo. Mundi Prensa México. 156 p.

Saavedra, A; Gutiérrez, S. 2014. Evaluación del efecto de tres sustratos en la producción de marango a nivel de vivero en la Universidad Nacional Agraria. Tesis de grado. Facultad de Ciencias Animales. Managua, NI. 67 p.

Sáenz R, JT; Villaseñor R, FJ; Muñoz F, HJ; Rueda, SA; Prieto R, JA. 2010 Calidad de plantas en viveros forestales de clima templado en Michoacán. Instituto de investigaciones forestales, agrícolas y pecuarias. Folleto técnico No.17. Michoacán, ME. 43 pp. 